\title{
Monolithically integrated dual-output DEML for full duplex DPSK-ASK and DPSK-SSB ONU for ultra-dense channel spaced access network
}

\author{
Guang Yong Chu, Victor Polo, Iván N. Cano, Christophe Kazmierski, Romain Brenot, and Josep Prat, \\ Member, IEEE
}

(Invited Paper)

\begin{abstract}
Ultra dense access network draws people's attention recently; however, until now it is difficult to implement it commercially owing to cost, footprint, and the traditional complexity of coherent receiver. Single laser based ONU provides a potential system for simplifying the ONU. Here we show a 2.5 Gb/s/user bidirectional transmission with $5 \mathrm{GHz}$ channel spacing between uplink and downlink for UDWDM. A monolithic integration on dual output DEML based ONU for ' $\lambda$-to-the-user' network is demonstrated. Furthermore, bidirectional DPSK-ASK and DPSK-SSB transmission with low-footprint, monolithically integrated DEML at ONU are also tested.
\end{abstract}

Index Terms-Dual output DEML, monolithically integrated chip, ultra dense access network, full duplex, low footprint ONU.

\section{INTRODUCTION}

$I^{n}$ n order to adapt the future network for $5 G$ Infrastructure Public Private Partnership (5G-PPP), the optical communications, in particular optical access network, requires to be refreshed [1]. Compared with the standard 10-GigabitCapable Passive Optical Network (XGPON) [2] and NextGeneration Passive Optical Network 2 (NGPON2) networks [35], ultra dense wavelength division multiplexed passive optical network (UDWDM-PON) [1,6] has the advantages of increased capacity per user and more number of the wavelength, providing a promising option of 'each user one wavelength'. Hence, the UDWDM-PON becomes one candidate for next generation PONs, such as NGPON3. The key characteristics of a UDWDM-based system are well beyond current and other future-planned PON based systems, and the key enabler is coherent detection for the function of wavelength selectivity instead of optical filtering.

Manuscript received 10 Oct., 2015. This work was supported by the European FP7 COCONUT Project (GA318515), PhD fellowship donated by China Scholarship Council (201207040059), and the Ministry of Science and Innovation under grant TEC2011-25215 (ROMULA Project).

Guang Yong Chu, Victor Polo, Iván N Cano, and Josep Prat are with the Department of Signal Theory and Communication, Universitat Politècnica de
Recently, colorless optical network unit (ONU) is applied with wavelength reusing scheme $[9,10]$ and seeding techniques $[11,12]$ with an optical multiplexing element. Differently, single laser based ONU $[1,7,8]$ could be also one solution for ONU, which increases the power budget for UDWDM-PON and shares the same optical source both for downstream and upstream as shown in Fig. 1(a), as downlink's local oscillator laser (LO) and uplink's carrier, respectively. The UDWDMPON scenario for single integrated laser based ONU is shown in Fig. 1(a), and a $12.5 \mathrm{GHz}$ ultra-dense channel spacing spectrum is shown in Fig. 1(b).

The ONU's footprint for next generation ultra-dense access networks is a vital factor to be considered [1,7]. Unlike the traditional large footprint $\mathrm{LiNbO}_{3}$ mach-zehnder architecture, monolithic integration on $\operatorname{InP}$ is a way to lower footprint, consumption, and to facilitate the design of complex photonic circuits with multiple functions [13].

In recent years, integrated photonics components are available for WDM-PON [1,7], such as reflective semiconductor optical amplifiers (RSOA) [10,14], binary phase shift keying electro-absorption modulated laser (BPSK-EML) [13], and dual electro-absorption modulated laser (DEML) [15]. In order to simplify the ONU with integrated components and extend this application for UDWDM-PON with coherent detection, the single integrated transmitter based ONU is desired.

We have demonstrate for the first time the DEML as dualoutput integrated chip for UDWDM-PON in [16]. Here is an extended version of [16]. In this work, a $2.5 \mathrm{~Gb} / \mathrm{s}$ differentially phase shift keying-amplitude shift keying (DPSK-ASK) and DPSK-single side band (DPSK-SSB) full duplex transmission using single integrated transmitter at ONU with heterodyne detection are tested; furthermore, the receiver at ONU uses only

Catalunya, 08034 Barcelona, Spain (e-mail: yong@tsc.upc.edu, polo@tsc.upc.edu, ivan.cano@tsc.upc.edu, jprat@tsc.upc.edu).

Christophe Kazmierski and Romain Brenot are with the III-V lab, a joint lab of 'Alcatel-Lucent Bell Labs France', 'Thales Research and Technology' and 'CEA Leti', Route de Nozay, 91460 Marcoussis, France. (romain.brenot@35lab.fr, christophe.kazmierski@3-5lab.fr). 


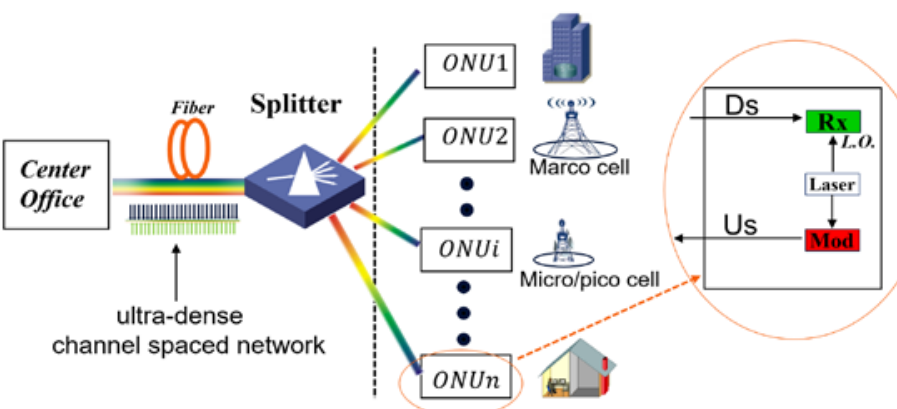

(a)

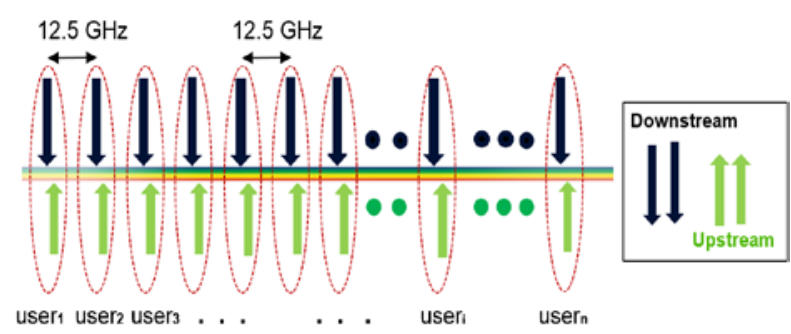

(b)

Fig. 1: (a) The application scenario for single laser based ONU for UDWDM-PON. (Rx: receiver, Mod: modulator, Ds: downstream, Us: upstream); (b)

The spectrum spacing of $12.5 \mathrm{GHz}$ access network.

one photo detector and achieves a high sensitivity both at ONU and optical line terminal (OLT). All above gives evidence for supporting the new effective structure with single integrated DEML at ONU for ultra-dense channel spaced network. The paper is organized as follows. In section II, the dual output DEML is described. The full duplex transmission experiment is defined in section III. The bidirectional DPSK-ASK system is shown in section IV. In section V, a full duplex DPSK-SSB system is presented. Finally, we summarize the work in section VI.

\section{DESCRIPTION OF DUAL-OUTPUT DEML}

The dual-output DEML is based on AlGaInAs-QW (quantum well) material for its large electronic confinement providing enhanced electro-absorption properties and reduced thermal carrier leakage; it emits in C-band at a wavelength of $1537 \mathrm{~nm}$. The same active layer is used for both laser (DFB) and modulator (EAM) sections. The length of the DFB and EAM sections are around $470 \mu \mathrm{m}$ and $75 \mu \mathrm{m}$ respectively. A schematic representation of DEML chip is shown in Fig. 2(a). The III-V epitaxial layers are grown on an InP substrate. Its intrinsic layer contains an AlGaInAs multiple-QW $(M Q W)$ stack sandwiched by two separate confinement heterostructures $(\mathrm{SCH})$ [17]. The EAM is implemented by using a pin diode structure with the active $M Q W$ located inside the intrinsic layer.

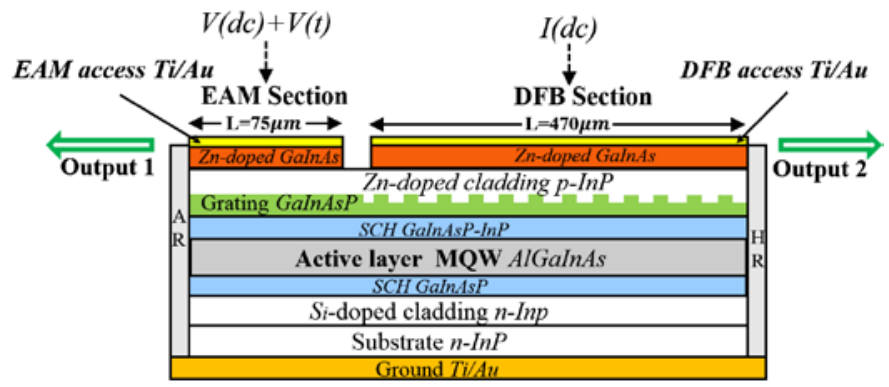

(a)

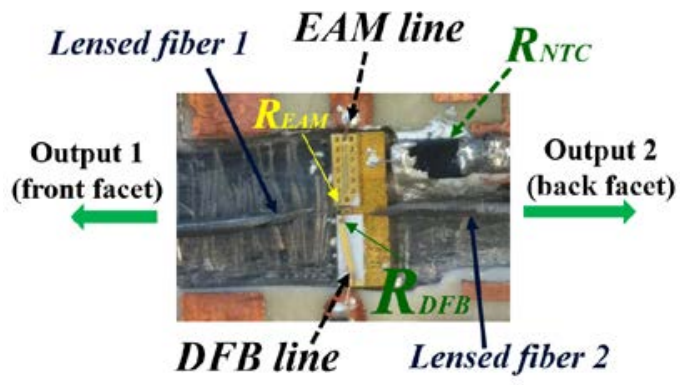

(b)

Fig. 2: (a) side-view of DEML (b) assembled 2-output-DEML

The bias applied to the pin diode adjusts the electrical field in the $M Q W$ region and results in the change of optical absorption due to the quantum-confined stark effect (QCSE). In addition, the waveguide is selectively buried with a tandem layer of semi-insulating $\mathrm{InP}$. The semi-insulating buried structure assures low EAM capacitance and low thermal resistance of the laser $[18,19]$.

The DEML chip (size $0.25 \mathrm{~mm} \times 0.5 \mathrm{~mm}$ ) is on a sub-mount (size $2 \mathrm{~mm} \times 6 \mathrm{~mm} \times 0.5 \mathrm{~mm}$ ), and RF data access ceramics for DFB and EAM are shown in Fig. 2(b). The assembled setup shown includes two lensed fiber at the both sides, one for EAM and another for DFB. A temperature sensor is placed beside of the chip. The DEML provides two output: one is the DFB output (back facet), another one for EAM output (front facet). Both the DFB and the EAM have resistors ( $\mathrm{R}_{\mathrm{EAM}}$ and $\mathrm{R}_{\mathrm{DFB}}$ ) of $50 \Omega$ for effective matched impedance.

In order to modulate the EAM, we firstly measured the characteristics of the DEML in the front facet. A low-couplingloss lensed fiber is used for the front facet of the chip. As shown in the Fig. 3, the DFB has a threshold as low as $11 \mathrm{~mA}$, the coupled power can reach as high as $3.2 \mathrm{~mW}$ when DFB launched at $100 \mathrm{~mA}$ thanks to the benefit of low-coupling-loss. DFB is operated at $70 \mathrm{~mA}$, a balanced value of temperature stability, maximum bandwidth and output power. From Fig. 3, the bias condition of $-2.4 \mathrm{~V}$ (linear region) is chosen, providing $-1 \mathrm{dBm}$ output power. 


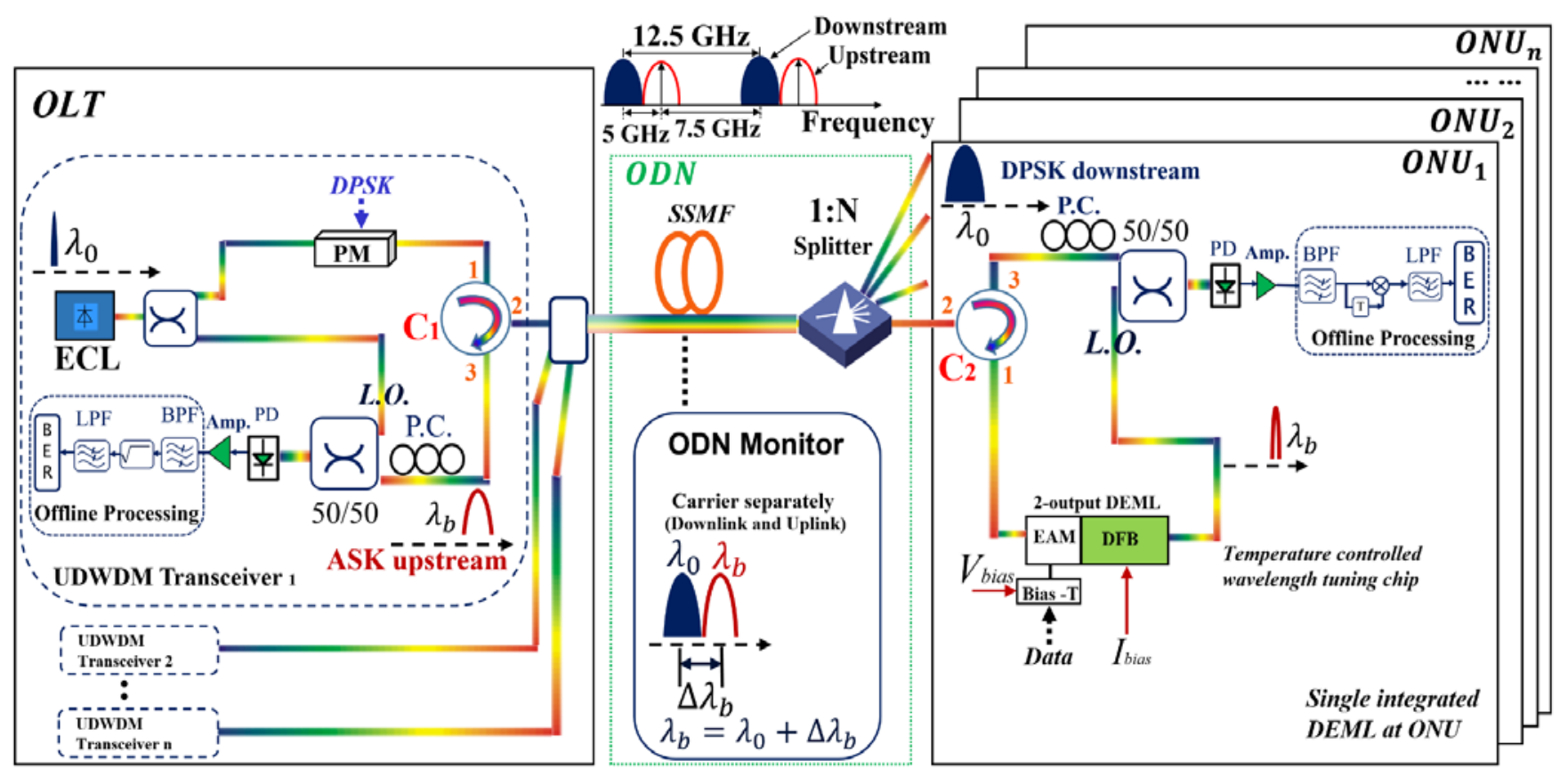

Fig. 4: Monolithically integrated dual-output-DEML based ONU for $2.5 \mathrm{~Gb} / \mathrm{s}$ bidirectional coherent UDWDM-PON (ODN: optical distribution network)

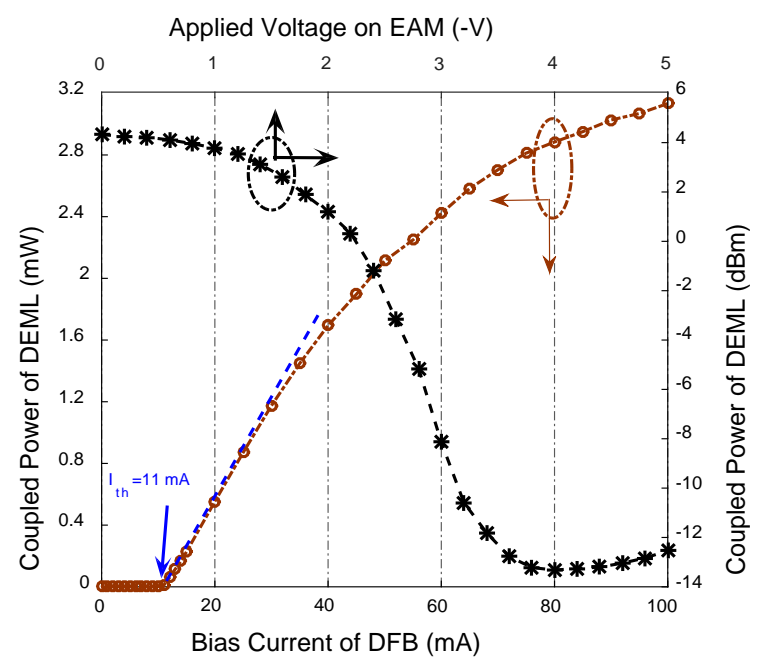

Fig. 3: The characterization of the DEML's front facet. (The front facet coupled power versus $\mathrm{DFB}$ bias current (right color, $\mathrm{V}_{\mathrm{EAM}}=0$ ), and the front facet power versus $\mathrm{EAM}$ bias voltage (black color, $\mathrm{I}_{\mathrm{DFB}}=70 \mathrm{~mA}$ ).

\section{SYSTEM SETUP}

The architecture of the proposed ultra-dense access network is shown in Fig. 4. The OLT consists of several UDWDM transceivers coupled, and each transceiver corresponds one ONU. The transceiver at OLT shares the same optical cource both for transmitter and coherent receiver. The signal are firstly generated from OLT, then after transmission over the feeder fiber, a power splitter distributes all wavelengths to the ONUs; heterodyne detection takes the benefits of the high sensitivity and the fine selection for the wavelength. Monolithically integrated dual-output-DEML is used both for the downstream's LO and upstream's optical source, which shows a super simplified ONU. The whole access network is suitable for 12.5 GHz channel spaced transparent network, not requiring optical filtering to select the wavelength for each ONU, thus being compatible with currently deployed PON distribution networks [20-22]. The polarization controller at ONU can be removed by following ways, such as the proposed common polarization scrambler [22], traditional polarization controller or using diversity.

At OLT, an external cavity laser (ECL) emitting at around $1537 \mathrm{~nm}$, is split with a coupler, serving the optical power both for downstream's modulator and upstream's LO.

For the downstream, a total of $2^{18}$ bits consisting of nonreturn to zero (NRZ) binary sequences are differentially encoded and modulate the phase modulator at $2.5 \mathrm{~Gb} / \mathrm{s}$. The electrical data is amplified before modulation to obtain $180^{\circ}$ phase variations, obtaining a DPSK signal. The optical distribution network is composed of a $50 \mathrm{~km}$ standard single mode fiber (SSMF) and splitter. The DFB section of the integrated DEML is employed at ONU, and is tuned at $5 \mathrm{GHz}$ shifted against the downstream carrier, which provides an intermediate frequency to perform the DPSK heterodyne detection.

The uplink transmission uses the same DFB as the optical carrier, but modulates the EAM section generating ASK optical signal. A $2.5 \mathrm{~Gb} / \mathrm{s}$ NRZ data is generated and operated in the EAM section. After the distribution network, at the OLT the upstream signal is coherently detected using heterodyne detection with a single PD.

For the experiments, firstly the DPSK and differential demodulation for downstream, and enveloped demodulation ASK for upstream are tested. Then SSB for upstream, and DPSK for downstream bidirectional transmission are also obtained. 


\section{BIDIRECTIONAL DPSK-ASK TRANSMISSION}

\section{A. Unidirectional transmission for DPSK downstream}

The $\mathrm{LiNbO}_{3}$ phase modulator is modulated at OLT and the signal is received coherently at the ONU as shown in the experimental setup in Fig. 4.

The DFB section of the DEML has a threshold as low as 11 $\mathrm{mA}$, and the back facet provides $-9 \mathrm{dBm}$ optical power for downstream's LO, and the limited power is restricted by the high reflection (Fig. 2(a)) from the back facet.

The bit error ratio (BER) against received input power is shown in Fig. 5; the sensitivity for $2.5 \mathrm{~Gb} / \mathrm{s}$ at $\mathrm{BER}=10^{-3}$ reaches $-35 \mathrm{dBm}$ and $-34.2 \mathrm{dBm}$ for back-to-back (BtB) and 50 $\mathrm{km}$ transmission, respectively. The $2.5 \mathrm{~Gb} / \mathrm{s}$ signal spectrum from electrical spectrum analyser (ESA) is shown in the inset.

The output power of DEML's back facet can be increased later by reducing the reflection, and $6 \mathrm{~dB}$ sensitivity improvement would be expected when $0 \mathrm{dBm}$ is provided, according to simulations.

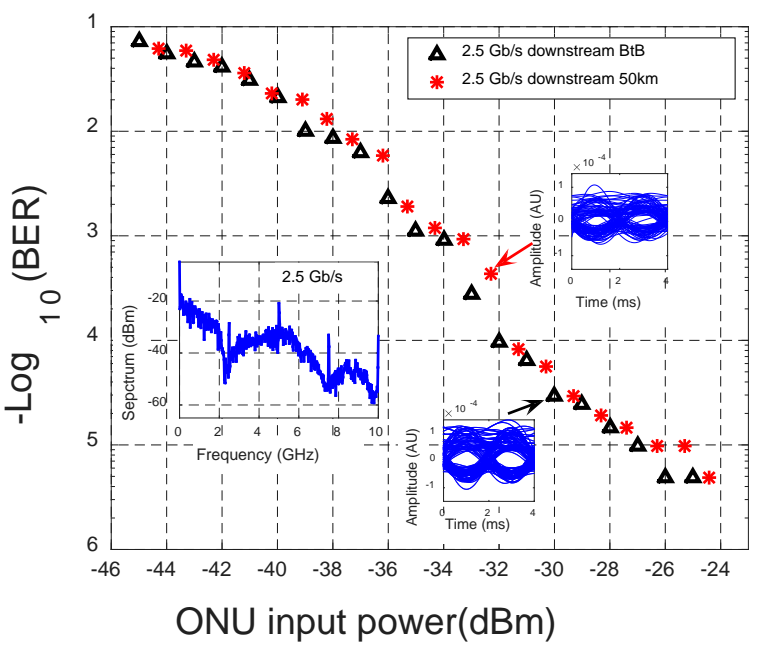

Fig. 5: Downstream BER versus received input power

\section{B. Unidirectional transmission for ASK upstream}

For transmitting data, the EAM section is modulated at the bias condition of $-2.4 \mathrm{~V}$ with a peak-to-peak signal amplitude of $1 \mathrm{~V}$. The frequency offset between the signal and the $\mathrm{LO}$ is $5 \mathrm{GHz}$. Negligible difference could be found between the BtB and $50 \mathrm{~km}$ transmission as shown in Fig. 6. The eye diagram and the signal spectrum are also shown in the inset of Fig. 6. The sensitivity reaches $-40.5 \mathrm{dBm}$ and $-39.7 \mathrm{dBm}$ for $\mathrm{BtB}$ and $50 \mathrm{~km}$, respectively.

The LO at OLT from the ECL is $0 \mathrm{dBm}$, which is $6 \mathrm{~dB}$ higher than the output power from back facet of the DEML, and $9 \mathrm{~dB}$ higher than the LO at ONU for the downstream transmission. This explains the reason why the sensitivity is much higher than the case of downstream.

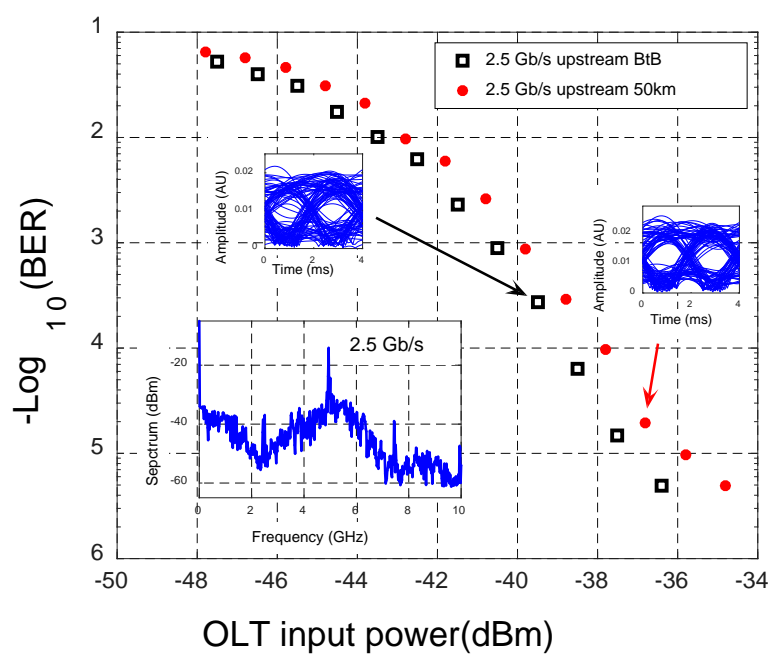

Fig. 6: Upstream BER versus received input power

\section{Full duplex DPSK-ASK transmission}

Bidirectional transmission described in Fig. 4, putting downstream and upstream systems in operation simultaneously, and is also evaluated as shown in Fig. 7. The coherent ASK can allow more phase noise tolerance than coherent DPSK [23-25], which explains that the downstream presents more influences on BER floor. What is more, the $\mathrm{LO}$ for downstream is $-9 \mathrm{dBm}$, much lower than the $\mathrm{LO}$ for upstream $(0 \mathrm{dBm})$, which explains that the upstream performance is better than the case of downstream.

At $\mathrm{BER}=10^{-3}$, the full duplex downstream sensitivity now reaches $-34.1 \mathrm{dBm}(\mathrm{BtB})$, and $-32.4 \mathrm{dBm}(50 \mathrm{~km})$. There is 1.7 $\mathrm{dB}$ penalty between $\mathrm{BtB}$ and $50 \mathrm{~km}$ fiber transmission, which is $0.9 \mathrm{~dB}$ larger than the case of unidirectional transmission. Less than $1 \mathrm{~dB}$ penalty is found compared with unidirectional transmission (Fig. 5) for back-to-back, and $50 \mathrm{~km}$ transmission has a BER floor both from the increasing phase noise and the backscattering effects.

The upstream transmission, the sensitivity reaches -39.8 $\mathrm{dBm}$ and $-38 \mathrm{dBm}$ for $\mathrm{BtB}$ and $50 \mathrm{~km}$ transmission, respectively. Compared with unidirectional case, there is $0.7 \mathrm{~dB}$ and $1.7 \mathrm{~dB}$ penalty for $\mathrm{BtB}$ and $50 \mathrm{~km}$ at $\mathrm{BER}=10^{-3}$; the backscattering at the overlapping spectrum slightly degrades the performance especially for the full duplex $50 \mathrm{~km}$ transmission.

The results show the successfully transmission with $5 \mathrm{GHz}$ channel spacing for downstream and upstream. The optical spectrum is detected using a high resolution optical spectrum analyser as the Fig. 7(a) and the inset of Fig. 7(b), which shows a $5 \mathrm{GHz}$ channel spacing between DPSK downstream and ASK upstream. The eye diagrams of downstream and upstream are shown in the inset of Fig. 7(b). 


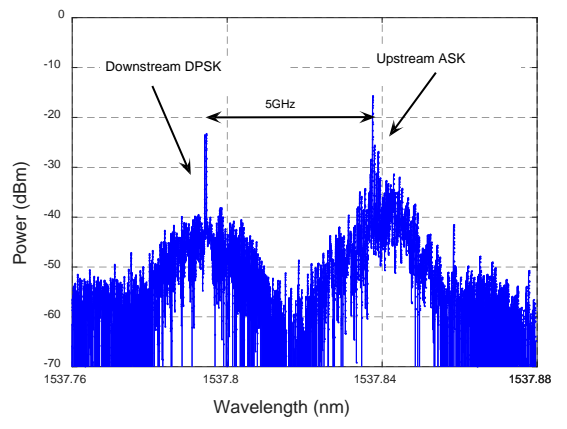

(a)

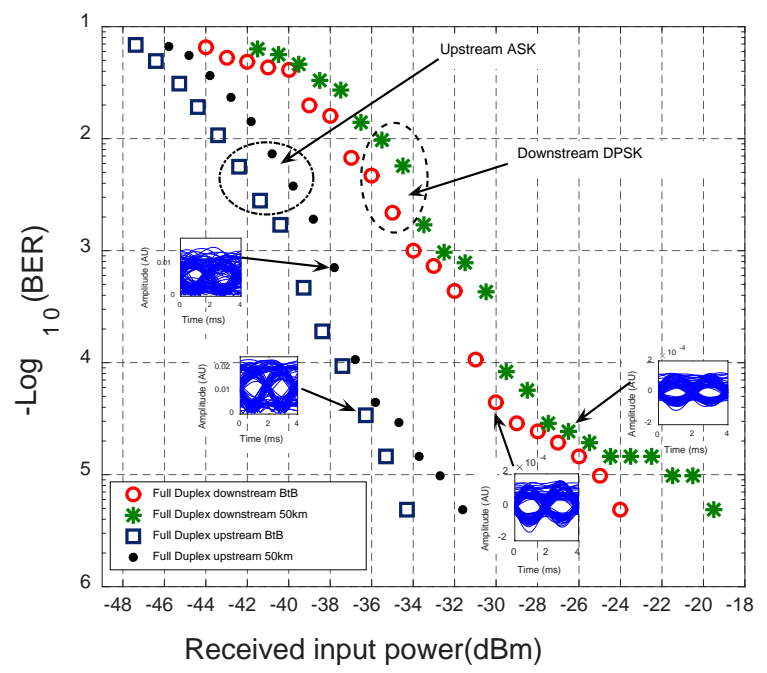

(b)

Fig. 7: (a) DPSK-ASK bidirectional spectrum (b)DPSK-ASK bidirectional transmission

\section{BIDIRECTIONAL DPSK-SSB TRANSMISSION}

\section{A. Single side band generation by DEML for upstream}

As explained in the previous sections, the UDWDM-PON has the feature of ultra-narrow channel spacing, providing more users; such as $25 \mathrm{GHz}, 12.5 \mathrm{GHz}$, or even $6.25 \mathrm{GHz}$. These ultra-narrow channel spacing requires the signal spectrum bandwidth as narrow as possible; based on this reason, the single side band has investigated in this DEML.

In order to give a proof of concept, here we firstly use a discrete-time signal, the sinusoidal signal is set as $500 \mathrm{MHz}$, and signal amplitude of $1 \mathrm{~V}_{\mathrm{pp}}, 2.4 \mathrm{~V}_{\mathrm{pp}}$ are separately provided into the EAM section. The signal spectrums are then directly captured using a high resolution optical spectrum analyzer (HROSA).

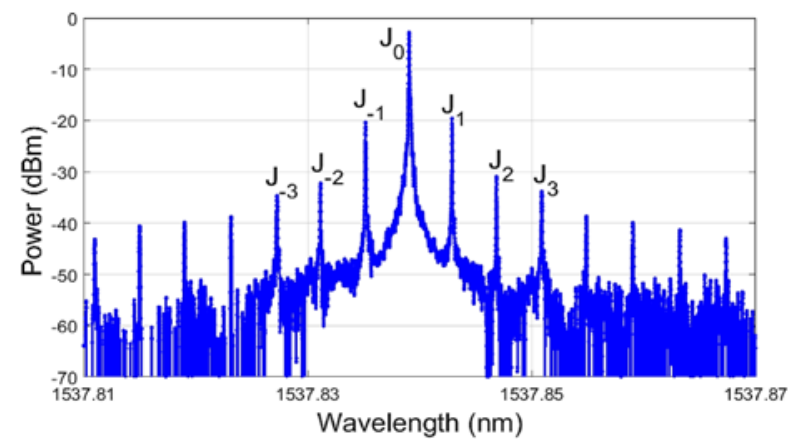

(a)

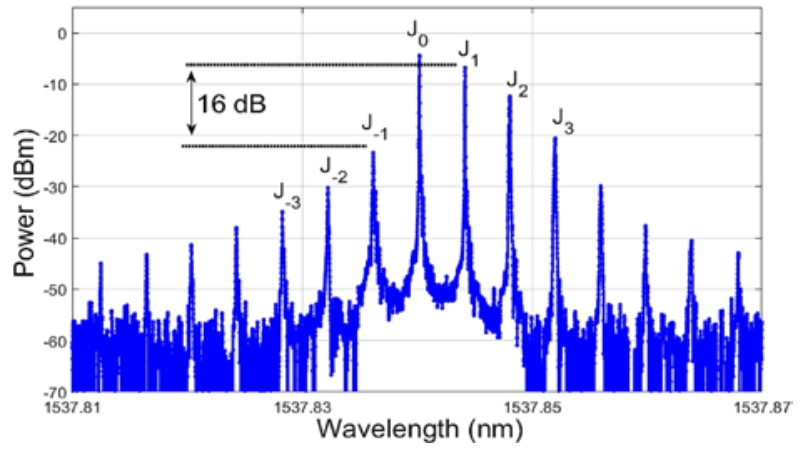

(b)

Fig. 8: (a) discrete-time signal for ASK mode with signal amplitude of 1 Vpp (b) discrete-time signal for ASK mode with signal amplitude of 2.4 Vpp.

As shown in Fig. 8(a), the spectrum shows no single side band generation; when increasing the amplitude to $2.4 \mathrm{Vpp}$, there is single side band generation. The side band ratio is 16 $\mathrm{dB}$ as shown in Fig. 8(b).

Then, we apply this signal amplitude to EAM, and modulating the signal in OSSB condition. So, the EAM section is modulated at the bias condition of $-2.4 \mathrm{~V}$ with a peak-to-peak signal amplitude of $2.4 \mathrm{~V}_{\mathrm{pp}}$. After fiber distribution, finally the signal is coherently detected at OLT. The frequency offset between the signal and the $\mathrm{LO}$ is $5 \mathrm{GHz}$. The BER performances for BtB and 50km transmission are shown in Fig. 9 . The eye diagram and the signal spectrum are also shown in the inset of Fig. 9. The sensitivity reaches at $-43.7 \mathrm{dBm},-42.4$ $\mathrm{dBm}$ for $\mathrm{BtB}$ and $50 \mathrm{~km}$, respectively. The LO at OLT from the ECL is maintained the same as the previous condition of $0 \mathrm{dBm}$.

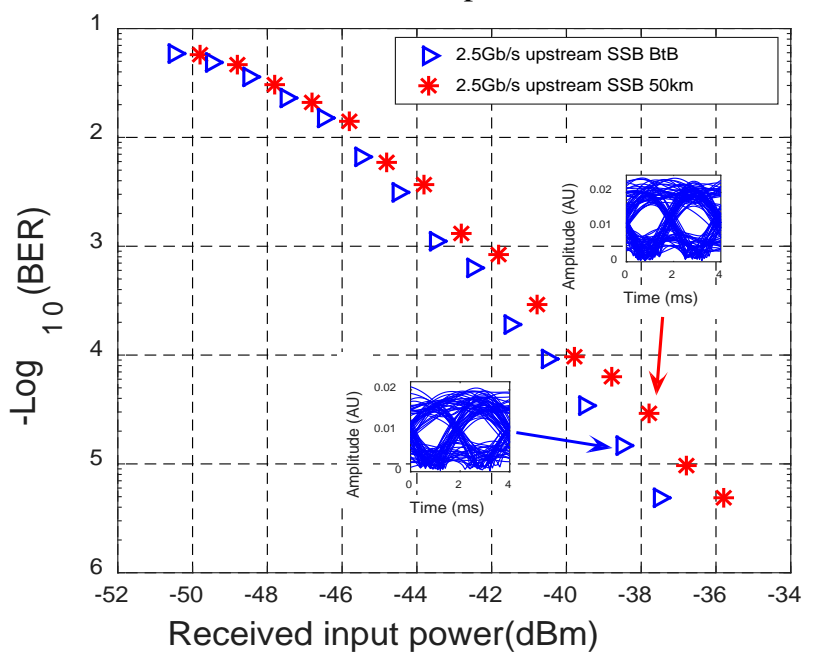

Fig. 9: Upstream SSB BER versus received input power

\section{B. Full duplex DPSK-SSB transmission}

Bidirectional transmission for DPSK-SSB presented in Fig. 3, is also evaluated as shown in Fig. 10. Similarly to the DPSKASK case, for full duplex transmission the coherent upstream SSB can allow more phase noise tolerance than coherent DPSK downstream, which explains that the downstream presents more influences on error floor. Besides, the $\mathrm{LO}$ for downstream is -9 $\mathrm{dBm}$, much lower than the LO for upstream $(0 \mathrm{dBm})$, which explains that the sensitivity for upstream is better than 
downstream.

For the full duplex downstream, the sensitivity for $2.5 \mathrm{~Gb} / \mathrm{s}$ at $\mathrm{BER}=10^{-3}$ reaches $-33 \mathrm{dBm}$ and $-31.5 \mathrm{dBm}$ for $\mathrm{BtB}$ and 50 $\mathrm{km}$ transmission, respectively. Compared with unidirectional transmission (previous section IV, Fig. 5), there is only around $2 \mathrm{~dB}$ penalty for back-to-back, and $50 \mathrm{~km}$ transmission, and there is higher error floor because of the increasing phase noise and the backscattering effects.

The bidirectional upstream SSB transmission, the sensitivity reaches $-42.7 \mathrm{dBm}$ and $-41.6 \mathrm{dBm}$ for $\mathrm{BtB}$ and 50 $\mathrm{km}$ transmission, respectively. Compared with unidirectional case, there is $1 \mathrm{~dB}$ and $1.2 \mathrm{~dB}$ penalty for $\mathrm{BtB}$ and $50 \mathrm{~km}$ at $\mathrm{BER}=10^{-3}$, the backscattering degrades the performance especially for the full duplex $50 \mathrm{~km}$ transmission.

The optical spectrum is detected using a high resolution optical spectrum analyser as the inset of Fig. 10(a), which shows a $5 \mathrm{GHz}$ channel spacing between DPSK downstream and SSB upstream. The eye diagrams of downstream and upstream are shown in the inset of Fig. 10(b).
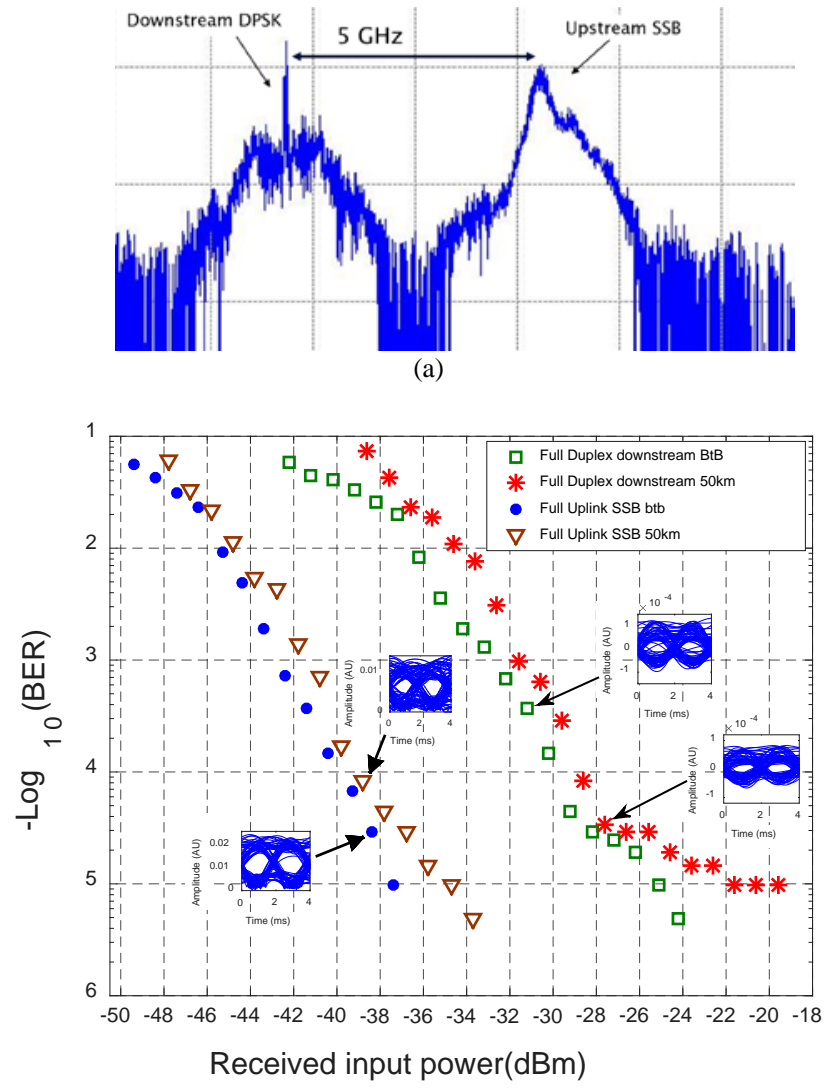

(b)

Fig. 10: (a) DPSK-SSB bidirectional spectrum (b) DPSK-SSB bidirectional transmission performances.

\section{CONCLUSION}

First demonstration with dual output DEML, and first implementation on single-integrated-chip based bidirectional UDWDM-PON ONU. A low footprint, cost-effective ONU is demonstrated for Ultra-Dense WDM-PON, with single monolithically integrated dual-output-DEML both as the transmitter and LO. Two scenario of $2.5 \mathrm{~Gb} / \mathrm{s} /$ user DPSK-ASK and DPSK-SSB bidirectional transmission, enabling a channel spacing at $12.5 \mathrm{GHz}$ between users, is tested successfully. Extremely simplified structure for both transmitter and receiver, consisting of single $\mathrm{PD}$ and single integrated laser chip, is used at ONU and achieves high sensitivity costeffectively. Besides, the SSB is generated with only injecting data via EAM section, and achieve as high as $16 \mathrm{~dB}$ side band ratio. Furthermore, at the SSB condition, the receiver sensitivity is $2 \mathrm{~dB}$ better than the case of ASK condition, both for unidirectional and bidirectional transmission, mainly because of higher signal amplitude. The ice on the cake is, the SSB can allow the channel spacing increase to super-dense access network, such as $6.25 \mathrm{GHz}$ spaced network including both downstream and upstream. All in all, the ONU is suitable for the future UDWDM-PON.

\section{REFERENCES}

[1] J. Prat, "Technologies for a Cost-effective Coherent udWDM-PON," Proc. OFC, Th3I, Los Angeles, 2015.

[2] 10-Gigabit-Capable Passive Optical Network (XG-PON), ITU-T G.987 Series Recommendations.

[3] 40-Gigabit-Capable Passive Optical Networks (NG-PON2), ITU-T G.989 Series Recommendations.

[4] D. Nesset, "NG-PON2 Technology and Standards," IEEE J. Lightw. Technol., vol. 33, no. 5, pp. 1136-1143, Mar. 2015.

[5] Y. Luo, X. Zhou, F. Effenberger, X. Yan, G. Peng, Y. Qian, and Y. Ma, "Time- and wavelength-division multiplexed passive optical network (TWDM-PON) for next-generation PON stage 2 (NG-PON2)," IEEE J. Lightw. Technol., vol. 31, no. 4, pp. 587-593, Feb. 2013.

[6] M. Presi, R. Corsini, M. Artiglia, and E. Ciaramella, "Ultra-dense WDMPON 6.25 GHz spaced $8 \times 1 \mathrm{~Gb} / \mathrm{s}$ based on a simplified coherent-detection scheme," Opt. Express, vol. 23, no. 17, pp. 22706, Aug. 2015.

[7] G. Y. Chu, V. Polo, A. Lerín, J. Tabares, I. N. Cano, J. Prat, “1.25-3.125 $\mathrm{Gb} / \mathrm{s}$ per user PON with RSOA as phase modulator for statistical wavelength ONU”, Optics Communications, vol. 357, pp. 34-40, Dec. 2015.

[8] G. Y. Chu, A. Lerín, I. N. Cano, V. Polo, J. A. Tabares, J. Prat, “Exploiting RSOA for Uplink Transmission with Coherent Detection for Low Cost UDWDM-PON," Proc. ACPC, AF2B.1, Shanghai, 2014.

[9] M. Omella, A. Jimenez, G. Bosco, P. Poggiolini, J. Prat, "Non-linear function for a Gaussian photo-reception in standard IM/DD systems," Optical and Quantum Electronics, vol. 42, no. 3, pp. 165-178, 2010.

[10] E. Kehayas, B. Schrenk, P. Bakopoulos, J. A. Lazaro, A. Maziotis, J.Prat, H. Avramopoulos, "All-Optical Carrier Recovery with Periodic Optical Filtering for Wavelength Reuse in RSOA-based Colorless Optical Network Units in Full-Duplex 10Gbps WDM-PONs," Proc. OFC, OWG4, San Diego, 2010.

[11] R. Brenot, "Demystification of Self-Seeded WDM Access," Proc. OFC, W1J1, Los Angeles, 2015.

[12] F. Saliou, G. Simon, P. Chanclou, M. Brunero, L. Marazzi, P. Parolari, M. Martinelli, R. Brenot, A. Maho, S. Barbet, G. Gavioli, G. Parladori, S. Gebrewold, J. Leuthold, "Self-seeded RSOAs WDM PON field trial for business and mobile front haul applications," Proc. OFC, M2A2, Los Angeles, 2015.

[13] C. Kazmierski, D. Carrara, K. Lawniczuk, G. Aubin, J. Provost, and R. Guillamet, "12.5GB Operation of a Novel Monolithic $1.55 \mu \mathrm{m}$ BPSK Source Based on Prefixed Optical Phase Switching," Proc. OFC, OW4J8, Anaheim, 2013.

[14] G. Y. Chu, V. Polo, A. Leín, I. Cano, J. Prat, "Optimizing Reflective Semiconductor Optical Amplifier as Phase Modulator for Low Cost Colorless ONU with 3x3 Homodyne Detection,” Proc. ACPC, AF2B.5, Shanghai, 2014.

[15] A. Lerín, G. Y. Chu, V. Polo, I. Cano, J. Prat, “Chip Integrated DFB-EAM for Directly Phase Modulation Performance Improvement in UDWDMPON”, Proc. ECOC, P.7.10, Valencia, 2015.

[16] G. Y. Chu, I. N. Cano, C. Kazmierski, R. Brenot, J. Prat, "First demonstration of monolithically integrated dual output DEML for fullduplex UDWDM-PON ONU”, Proc. ECOC, Th.1.3.1, Valencia, 2015.

[17] D. Erasme, T. Anfray, M. E. Chaibi, K. Kechaou, J. Petit, G. Aubin, K. Merghem, C. Kazmierski, J. Provost, P. Chanclou and C. A. Berthelemot, 
“The Dual-Electroabsorption Modulated Laser, a Flexible Solution for Amplified and Dispersion Uncompensated Networks over Standard Fiber,” IEEE J. Lightw. Technol.,VOL. 32, NO. 21, Nov.1, 2014.

[18] C. Kazmierski, "Electro-Absorption-Based Fast Photonic Integrated Circuit Sources for Next Network Capacity Scaling," IEEE/OSA J. Opt. Commun. Netw., vol. 4, pp. A8-A16, 2012.

[19] William S. C. Chang, RF Photonic Technology in Optical Fiber Links, 166-170, Chapter 6, Cambridge University Press, 2002.

[20] I. N. Cano, A. Lerín, V. Polo, and Josep Prat, "Direct Phase Modulation DFBs for Cost-Effective ONU Transmitter in udWDM PONs," IEEE Photon. Technol. Lett., vol. 26, no. 10, 973-975, 2014.

[21] V. Polo, P. Borotau, A. Lerin, J. Prat, "DFB Laser Reallocation by Thermal Wavelength Control for Statistical udWDM in PONs," Proc. ECOC, P.4.1.3, Cannes, 2014.

[22] I. N. Cano, A. Lerín, V. Polo, and J. Prat, "Polarization Independent Single-PD Coherent ONU Receiver with Centralized Scrambling in udWDM-PONs”, Proc. ECOC, P.7.12, Cannes, 2014.

[23] L. G. Kazovsky, S. Benedetto, and A. E. Willner, Optical fiber communication systems, 4th Ed., 388-390, London: Artech house, 1996.

[24] J. Prat, V. Polo, P. Zakynthinos, I. Cano, J. A. Tabares, J. M. Fàbrega, D. Klonidis, and I. Tomkos, Simple intradyne PSK system for udWDMPON, Opt. Express, vol. 27, no. 17, pp. 28758-28763, 2012.

[25] J.M. Fabrega and J. Prat, "Homodyne receiver prototype with timeswitching phase diversity and feedforward analog processing," Opt. Letters, vol. 32, no. 5, 2007. 